\title{
Nebulized dexmedetomidine-lidocaine inhalation as a premedication for flexible bronchoscopy: a randomized trial
}

\author{
Wei Gu, Meiying Xu, Huijie Lu, Qi Huang, Jingxiang Wu \\ Department of Anaesthesiology, Shanghai Chest Hospital, Shanghai Jiao Tong University, Shanghai 200030, China \\ Contributions: (I) Conception and design: J Wu; (II) Administrative support: J Wu, M Xu; (III) Provision of study materials or patients: W Gu, H Lu, \\ Q Huang; (IV) Collection and assembly of data: W Gu, H Lu, Q Huang; (V) Data analysis and interpretation: W Gu, J Wu, M Xu; (VI) Manuscript \\ writing: All authors; (VII) Final approval of manuscript: All authors. \\ Correspondence to: Jingxiang Wu, MD. Department of Anaesthesiology, Shanghai Chest Hospital, Shanghai Jiao Tong University, No. 241 Huaihai \\ Rd. West, Shanghai 200030, China. Email: wu_jingxiang@sjtu.edu.cn.
}

Background: Dexmedetomidine plus opioid infusion after topical anaesthesia with nebulized lidocaine for cough suppression is a commonly used method for flexible bronchoscopy. Recently, the use of dexmedetomidine as an additive to local anaesthetics has been reported to have several advantages over conventional intravenous administration. However, there are no data regarding the use of nebulized dexmedetomidine-lidocaine for topical anaesthesia as a premedication for flexible bronchoscopy. Therefore, this study compared the tolerability and safety of nebulized dexmedetomidine with that of conventional intravenous administration in patients undergoing bronchoscopy with moderate sedation.

Methods: Sixty patients requiring flexible bronchoscopy were randomly assigned to three groups: (I) nebulized dexmedetomidine + lidocaine, $\mathrm{n}=20$; (II) intravenous dexmedetomidine + nebulized lidocaine, $\mathrm{n}=20$; and (III) nebulized lidocaine alone (no dexmedetomidine), $\mathrm{n}=20$. The patients' coughing scores were assessed and graded. Our primary hypothesis was that nebulized dexmedetomidine-lidocaine could reduce the incidence of moderate to severe coughing. The secondary endpoints were the rates of glottis closure, complete jaw relaxation and limb movement during the procedure; the elapsed time until recovery; and the dosages of vasoconstrictors and atropine.

Results: The incidence of moderate to severe coughing was $15 \%$ in the nebulized dexmedetomidine group, $50 \%$ in the intravenous dexmedetomidine group and $55 \%$ in the no dexmedetomidine group. The nebulized dexmedetomidine group had the lowest incidence of moderate to severe coughing $(\mathrm{P}=0.019)$. Nebulized dexmedetomidine showed a protective effect for reducing coughing compared with intravenous dexmedetomidine [P=0.008, odds ratio (OR): 0.273, 95\% confidence interval (CI): 0.089-0.833]. No differences in the rates of complete jaw relaxation and limb movement during the procedure were observed among the three groups (all $\mathrm{P}>0.05)$. The rates of glottis closure were similar (20\%, 25\%, and 35\%; P>0.05). The elapsed time until recovery in the nebulized dexmedetomidine group was significantly shorter than that in the intravenous dexmedetomidine group $(10.60 \pm 1.39$ vs. $15.10 \pm 1.45, \mathrm{P}<0.001)$. The vasoconstrictor dosages were significantly lower in the nebulized dexmedetomidine group than in the intravenous dexmedetomidine group $(\mathrm{P}<0.001)$.

Conclusions: Nebulized dexmedetomidine-lidocaine inhalation as a premedication for flexible bronchoscopy was well tolerated during bronchoscopies performed under moderate sedation and was associated with a reduced incidence of moderate to severe coughing, a shorter recovery time and reduced vasoconstrictor consumption.

Keywords: Anaesthesia; flexible bronchoscopy; dexmedetomidine; coughing

Submitted Jun 01, 2019. Accepted for publication Sep 27, 2019.

doi: $10.21037 /$ jtd.2019.10.59

View this article at: http://dx.doi.org/10.21037/jtd.2019.10.59 


\section{Introduction}

Flexible bronchoscopy is a well-established diagnostic and therapeutic procedure for patients with a variety of respiratory diseases. To facilitate the procedure, reduce coughing, and increase patient tolerance and comfort, patients are usually under monitored anaesthesia care (MAC) during bronchoscopy (1). As the procedure is usually performed on an outpatient basis, the ideal pharmacokinetic characteristics of sedatives during MAC for flexible bronchoscopy are rapid onset, short duration, and rapid recovery. Intravenous infusion of propofol and remifentanil combined with dexmedetomidine is a common method. Dexmedetomidine is a highly selective adrenergic $\alpha-2$ agonist that does not cause respiratory depression and can be used as a sedative for various procedures $(2,3)$. The combination of dexmedetomidine plus opioids or propofol infusion plus topical anaesthesia with lidocaine for cough suppression is commonly used for flexible bronchoscopy (4). However, it has been reported that intravenous dexmedetomidine is associated with a longer recovery time and worse bronchoscopist satisfaction scores (5). This result can be explained by the fact that dexmedetomidine has no antitussive effects, and the passage of a flexible bronchoscope through the vocal cords can lead to coughing (5). Recently, the use of dexmedetomidine as an additive to local anaesthetics was reported to have several advantages over conventional intravenous administration $(6,7)$. Animal experiments have indicated that the local application of dexmedetomidine to the airway can expand the smooth muscle of the trachea and may inhibit the cough response (8). Nebulized dexmedetomidine combined with other anaesthetics as a premedication for outpatient paediatric dental procedures has also been reported to provide smoother induction of general anaesthesia with a more rapid recovery and no significant side effects (9). However, there are no data regarding the use of nebulized dexmedetomidine-lidocaine for endobronchial topical anaesthesia as a premedication for flexible bronchoscopy. Therefore, this study compared the tolerability and safety of nebulized dexmedetomidine with those of conventional intravenous dexmedetomidine administration. Our primary hypothesis is that nebulized dexmedetomidine-lidocaine could reduce the incidence of moderate to severe coughing. The secondary endpoints included the rates of glottis closure, complete jaw relaxation and limb movement during the procedure; the elapsed time until recovery; and the dosages of vasoconstrictors and atropine.

\section{Methods}

This study was approved by the Institutional Review Board of Shanghai Chest Hospital (IRB \#KS 1748), and written informed consent was obtained from each patient before enrolment. This trial was registered before subject enrolment began at chictr.org.cn (ChiCTR1800015558; principal investigator, Jingxiang $\mathrm{Wu}$; date of registration, April 07, 2018). Recruitment extended from April 2018 until September 2018.

Sixty-three patients requiring flexible bronchoscopy were assessed for eligibility. The inclusion criteria were as follows: (I) age older than 18 years, (II) American Society of Anesthesiologists (ASA) physical status of I-II, and (III) body mass index (BMI) $\leq 30 \mathrm{~kg} / \mathrm{cm}^{2}$. Patients were excluded if they had (I) clinically important coronary atherosclerotic heart disease, (II) a history of stroke or transient ischaemic attack, or (III) sinus bradycardia or heart block.

\section{Protocol}

All procedures were performed by the same team, which included a bronchoscopist, a nurse and an anaesthesiologist, all of whom were blind to the group assignment. The patients were unaware of treatment group allocation. Randomization and drug masking were completed by a specialist who was not directly involved in the trials. Patients inhaled the nebulized solution of $2 \%$ lidocaine in a semi-reclining position in the preparation room. A Pari LC Plus ${ }^{\circledR}$ nebulizer and Pari TurboBoy ${ }^{\mathrm{TM}}$ compressor (PARI TurboBOY, Starnberg, Germany) were used. Monitoring included electrocardiography, $\mathrm{SpO}_{2}$, and noninvasive blood pressure. The patients received $20 \mathrm{~mL}$ of masked solution (dexmedetomidine or normal saline (NS) depending on the group allocation) at an infusion rate of $60 \mathrm{~mL} / \mathrm{h}$. General anaesthesia was then induced with targetcontrolled infusion of propofol and remifentanil at plasma concentrations of $4 \mu \mathrm{g} / \mathrm{mL}$ and $4 \mathrm{ng} / \mathrm{mL}$, respectively. Then, a 4\# Ambu AuraOnce laryngeal mask (Ambu, Copenhagen, Denmark) was inserted and connected to the ventilator. Propofol administration was adjusted to target a bispectral index (Medtronic monitor, Minneapolis, USA) between 40 and 50 . Remifentanil was adjusted to $2 \mathrm{ng} / \mathrm{mL}$ during anaesthesia maintenance. After the bronchoscopy examination, the patients were transferred to the postanaesthesia care unit for extubation.

Patients were randomly assigned in a ratio of 1:1:1 to three groups based on a computer-generated randomization 
sequence. An opaque, sealed envelope was opened to determine each patient's group assignment after the patient provided written informed consent. The three groups were as follows: (I) nebulized dexmedetomidine + lidocaine, (II) intravenous dexmedetomidine + nebulized lidocaine, and (III) nebulized lidocaine alone (no dexmedetomidine). The total dosage of dexmedetomidine was $0.6 \mu \mathrm{g} / \mathrm{kg}$ in both the nebulized and intravenous groups. An equal volume of NS was used as a sham for dexmedetomidine, and the drug syringe was coded for application. Each group received a $10 \mathrm{~mL} 2 \%$ lidocaine mix solution for nebulization and a $20 \mathrm{~mL}$ drug solution for infusion. Dexmedetomidine was added to the $10 \mathrm{~mL}$ of $2 \%$ lidocaine mix solution in the nebulized dexmedetomidine group or to the $20 \mathrm{~mL}$ of drug solution for infusion in the intravenous dexmedetomidine group.

\section{Measurements}

The patients' cough scores and glottis status assessments were used to assess the procedural conditions for bronchoscopy. The primary endpoint was whether nebulized dexmedetomidine-lidocaine could reduce the incidence of moderate to severe coughing during bronchoscopy. The secondary endpoints included the rate of glottis closure, the rates of complete jaw relaxation and limb movement during the procedure, the elapsed time until recovery and the dosages of vasoconstrictors and atropine.

A flexible bronchoscope (BF-F260, Olympus, Tokyo, Japan) was inserted via a laryngeal mask with the subjects in the supine position. Upon visualizing the vocal cords, $60 \mathrm{mg}$ of $2 \%$ lidocaine was delivered through the bronchoscope channel for cough suppression; an additional $50 \mathrm{mg}$ was delivered upon visualization of the carina. Additional topical anaesthesia was instilled at the discretion of the bronchoscopists. The nurse, who was unaware of the type of medication given to the patients, recorded the number of coughing episodes, if any. The severity of coughing was graded based on the number of coughing episodes (mild, 1-2; moderate, 3-4; and severe, 5 or more) (10). Glottis status referred to the position of the vocal cords (open, closed). Other scores, such as jaw relaxation (relaxed, or rigid) and limb movements (none, obvious), were assessed by the anaesthesiologist, who was blinded to the group assignments. Intraoperative hypotension was defined as systolic blood pressure $\leq 90 \mathrm{mmHg}$ lasting $5 \mathrm{~min}$ on two non-invasive blood pressure measurement cycles; in such cases, ephedrine $(6 \mathrm{mg})$ was administered. The total intraoperative consumption of ephedrine was recorded. The outcome assessors and data analysts were blinded to the group assignments. The elapsed time until recovery was defined as the time between the cessation of anaesthetic administration and the removal of the laryngeal mask. If the patient had a persistent severe cough, $3 \%$ sevoflurane or $0.8 \mathrm{mg} / \mathrm{kg}$ rocuronium was used for rescue.

\section{Data analysis}

SPSS 22.0 (IBM, Chicago, IL, USA) was used for data analysis. The data are presented as the means $[ \pm$ standard deviations (SDs)], N (\%), or mean differences with confidence intervals. Chi-squared tests were used for categorical variables; any unbalanced factors were adjusted for in all analyses. Relative risk values and $95 \%$ confidence intervals (CIs) were calculated. Secondary outcomes, including the rate of glottis closure, the rates of complete jaw relaxation and limb movement during the procedure, the elapsed time until recovery and the dosage of vasoconstrictors, were reported descriptively. $\mathrm{P}$ values $<0.05$ were considered significant.

\section{Sample size consideration}

In a preliminary study, the incidence of moderate to severe coughing in the conventional group was $60 \%$, and a reduction from $60 \%$ to $15 \%$ was considered to be of clinical importance $(\alpha=0.05$, power $=0.8)$. The analysis showed that 17 subjects per group would be sufficient to detect a difference. Assuming a $10 \%$ drop-out rate, the final sample size was set at 20 patients per group.

\section{Results}

In all, 63 patients undergoing flexible bronchoscopy were screened between May 2018 and June 2018. Three patients were excluded because they did not meet the inclusion criteria: one had a history of stroke, one had a history of myocardial infarction, and one had sinus bradycardia. A total of 60 patients were randomized (Figure 1). Baseline characteristics were comparable among the three groups (Table 1).

The incidence of moderate to severe coughing was $15 \%$ in the nebulized dexmedetomidine group, $50 \%$ in the intravenous dexmedetomidine group and $55 \%$ in the no dexmedetomidine group. Nebulized dexmedetomidine had the lowest incidence of moderate to severe coughing 
CONSORT 2010 Flow Diagram

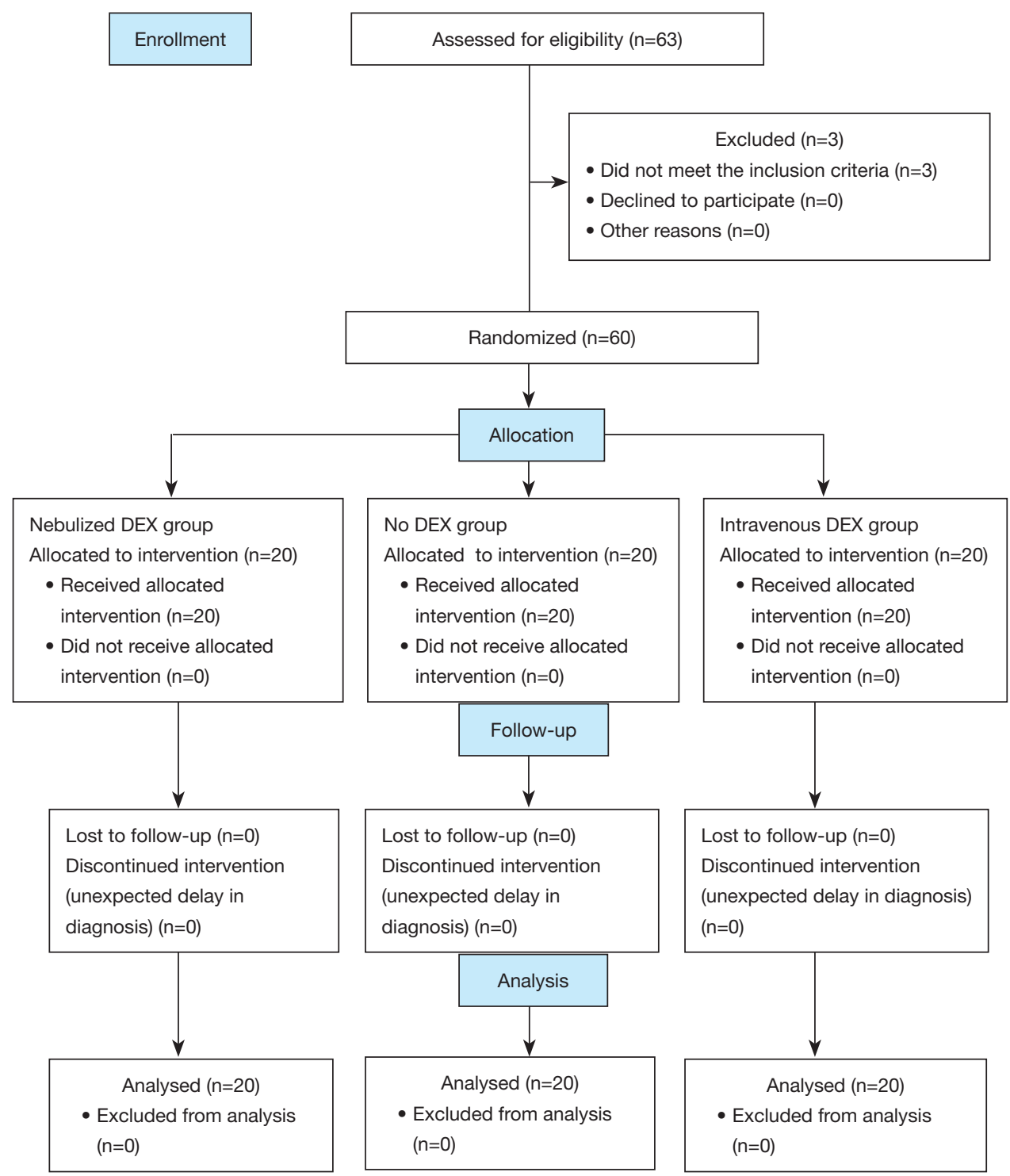

Figure 1 Flowchart of patients enrolled in the study. DEX, dexmedetomidine.

Table 1 Demographic characteristics and baseline among the 3 groups

\begin{tabular}{lccc}
\hline Variable & Nebulized DEX $(\mathrm{n}=20)$ & No DEX $(\mathrm{n}=20)$ & Intravenous DEX $(\mathrm{n}=20)$ \\
\hline Age (year) & $63.00 \pm 7.21$ & $58.70 \pm 9.07$ & $59.60 \pm 8.18$ \\
Male/female $(\mathrm{n})$ & $13 / 7$ & $11 / 9$ & $11 / 9$ \\
Weight $(\mathrm{kg})$ & $60.00 \pm 6.81$ & $61.70 \pm 7.42$ & $61.95 \pm 8.94$ \\
Height $(\mathrm{cm})$ & $163.45 \pm 5.73$ & $165.65 \pm 6.77$ & $164.90 \pm 8.80$ \\
ASA I/II (n) & $4 / 16$ & $5 / 15$ & $4 / 16$ \\
Duration of procedure (min) & $23.90 \pm 12.72$ & $24.90 \pm 12.17$ & $20.85 \pm 7.53$ \\
\hline
\end{tabular}

DEX, dexmedetomidine. 
Table 2 Incidence and severity of coughing among the 3 groups

\begin{tabular}{|c|c|c|c|c|c|c|}
\hline \multirow{2}{*}{ Variable } & \multicolumn{3}{|c|}{ Coughing, $n$ [\%] } & \multicolumn{3}{|c|}{ Coughing, $\mathrm{n}$ [\%] } \\
\hline & None & Slight & Total & Moderate & Severe & Total \\
\hline Nebulized DEX $(n=20)$ & $6[30]$ & $11[55]$ & $17[85]$ & $3[15]$ & $0[0]$ & $3[15]$ \\
\hline No DEX $(n=20)$ & $5[25]$ & 5 [25] & $10[50]$ & $8[40]$ & $2[10]$ & $10[50]$ \\
\hline Intravenous DEX (n=20) & $4[20]$ & $5[25]$ & $9[45]$ & $9[45]$ & $2[10]$ & $11[55]$ \\
\hline
\end{tabular}

DEX, dexmedetomidine.

Table 3 Condition of the vocal cords and jaw relaxation and limb movement among the 3 groups

\begin{tabular}{lcccc}
\hline Variable & Nebulized DEX $(\mathrm{n}=20)$ & No DEX $(\mathrm{n}=20)$ & Intravenous DEX $(\mathrm{n}=20)$ & P value \\
\hline Vocal cords, $\mathrm{n}[\%]$ & & & & 0.551 \\
Open & $16[80]$ & $15[75]$ & $13[65]$ & 7 [35] \\
Closed & $4[20]$ & $5[25]$ & & 0.909 \\
Jaw relaxation, $\mathrm{n}[\%]$ & & $15[75]$ & 5.641 \\
Complete & $16[80]$ & $5[25]$ & $5[25]$ & \\
Rigid & $4[20]$ & & $15[75]$ & \\
Limb movement, $\mathrm{n}[\%]$ & & $17[85]$ & $5[25]$ \\
None & $17[85]$ & $3[15]$ & & \\
Severe & $3[15]$ & &
\end{tabular}

DEX, dexmedetomidine.

$(\mathrm{P}=0.019)$. Sevoflurane inhalation was used as a remedy for severe coughing in 2 cases each in the intravenous dexmedetomidine and no dexmedetomidine group. Nebulized dexmedetomidine had a protective effect for coughing reduction compared with intravenous dexmedetomidine ( $\mathrm{P}=0.008$, OR: 0.273 , 95\% CI: 0.089 0.833) (Table 2).

The rates of glottis closure were similar among the three groups $(20 \%, 25 \%, 35 \% ; \mathrm{P}>0.05)$. There were no significant differences in the rates of complete jaw relaxation and limb movement during the procedure among the three groups (all $\mathrm{P}>0.05$ ) (Table 3).

The elapsed time until recovery in the nebulized dexmedetomidine group was significantly shorter than that in the intravenous dexmedetomidine group $(10.60 \pm 1.39 \mathrm{vs}$. $15.10 \pm 1.45, \mathrm{P}<0.001$ ) (Figure $2 A$ ). The dosage of ephedrine was significantly lower in the nebulized dexmedetomidine group than in the intravenous dexmedetomidine group $(\mathrm{P}<0.001)$ (Figure 2B). The dosage of atropine was significantly lower in the nebulized dexmedetomidine group than in the intravenous dexmedetomidine group
$(\mathrm{P}=0.029)$ (Figure 2C).

\section{Discussion}

This randomized double-blind trial showed that nebulized dexmedetomidine-lidocaine inhalation as a premedication for flexible bronchoscopy was well tolerated during bronchoscopies under moderate sedation and was associated with a reduced incidence of moderate to severe coughing, a shorter recovery time and reduced vasoconstrictor consumption. The patients in the nebulized dexmedetomidine group had the lowest incidence of moderate to severe coughing $(15 \%$ vs. $50 \%$ vs. $55 \% ; \mathrm{P}=0.019)$ compared with those in the intravenous dexmedetomidine and no dexmedetomidine groups. Nebulized dexmedetomidine showed a protective effect against coughing compared with intravenous dexmedetomidine ( $\mathrm{P}=0.008$, OR: $0.273,95 \% \mathrm{CI}$ : 0.089-0.833).

The British Thoracic Society has published guidelines that recommend offering sedation to all patients undergoing flexible bronchoscopy (11). However, no 
A

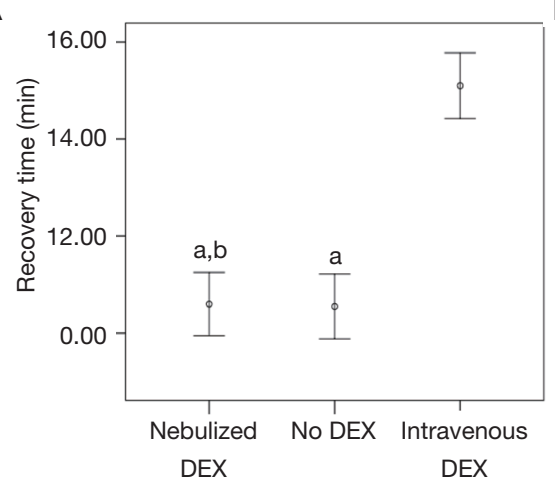

B

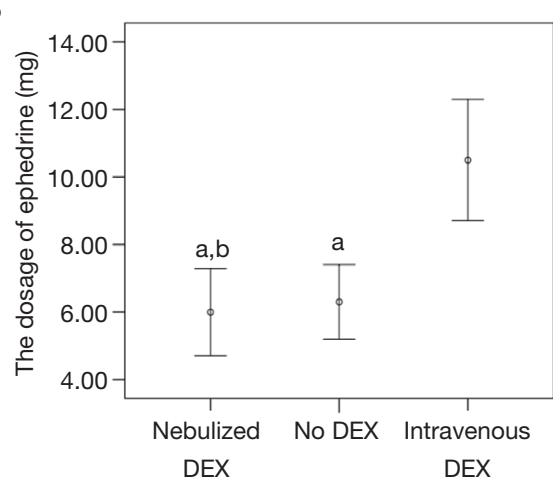

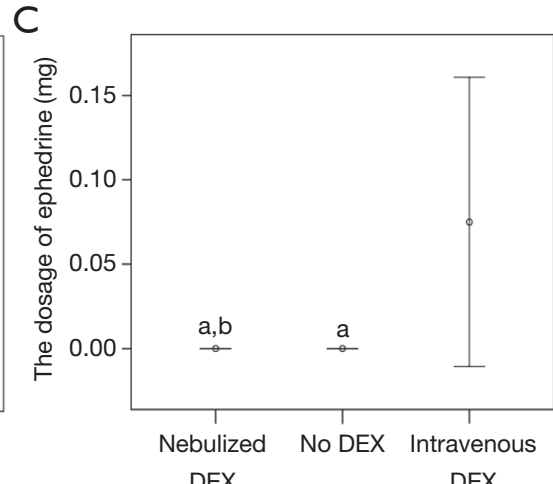

DEX

DEX

Figure 2 Recovery time (A), use of ephedrine (B) and use of atropine (C) among the 3 groups. All values are expressed as the mean \pm SD. ${ }^{\text {a }}$, $\mathrm{P}<0.05$ compared to intravenous dexmedetomidine. ${ }^{\mathrm{b}}, \mathrm{P}<0.05$ compared to no dexmedetomidine. Analysed by one-way ANOVA followed by LSD post hoc multiple comparison. DEX, dexmedetomidine.

standardized sedation protocol has been available until now, although an increasing number of studies have focused on searching for appropriate anaesthesia methods for flexible bronchoscopy (12-16). In its early years, flexible bronchoscopy was performed under general anaesthesia with muscle relaxation and ventilation through a tracheal tube or laryngeal mask. This method not only ensures the patient's oxygen supply but also reduces discomfort during the examination and provides better operative conditions for bronchoscopists (17). However, depolarizing muscle relaxants such as suxamethonium are likely to cause muscle pain, and non-depolarizing muscle relaxants may prolong the patient's waking time. An increasing number of recent studies have shown that MAC with laryngeal mask ventilation has unique advantages for outpatient flexible bronchoscopy management (18). It provides appropriate operating conditions for the bronchoscopist and accelerates recovery $(19,20)$. Intravenous infusion of propofol and remifentanil combined with dexmedetomidine has been used for flexible bronchoscopy in previous reports (13). However, the incidence of coughing during the procedure is still high (21). Dexmedetomidine has sedative, anxiolytic, and analgesic properties without causing respiratory depression $(2,3)$. Previous studies have reported that nebulized dexmedetomidine combined with other anaesthetics as a premedication for paediatric outpatients could provide smoother induction of general anaesthesia with more rapid recovery and no significant side effects (9). Therefore, the current trial compared the effect of nebulized dexmedetomidine inhalation with intravenous dexmedetomidine on cough inhibition during bronchoscopy.
Nebulization has not been recommended as a technique for delivering lidocaine to the airways because of the lack of determinate evidence comparing the need for additional doses of lidocaine and cough suppressant with doubling the total dose of lidocaine administered $(11,22)$. However, a recent study showed that endobronchial administration of lidocaine during bronchoscopy via a nebulizer was well tolerated and safe and was associated with reduced lidocaine and fentanyl dosages compared to administration via a syringe (21). Nebulized lidocaine also appeared to provide sufficient laryngopharyngeal anaesthesia for diagnostic transnasal tracheoscopy (23) and subjected the patients to fewer annoying manoeuvres (24). We still used nebulized lidocaine in the study and limited the total dosage to within the safe range.

The extravascular use of dexmedetomidine and its pharmacokinetics in humans have long been studied. Nebulized dexmedetomidine administration may allow rapid drug absorption through the nasal, respiratory, and buccal mucosa, which allows a bioavailability of $65 \%$ through the nasal mucosa and $82 \%$ through the buccal mucosa (25). Dexmedetomidine is colourless and odourless, does not stimulate the mucosa, has high bioavailability (25), and has direct actions on peripheral receptors, activating alpha 2 receptors, relaxing smooth muscles and dilating the bronchi, thereby reducing the cough reflex (26).

In our study, the incidence of moderate to severe coughing was significantly reduced from $55 \%$ to $15 \%$ by nebulized dexmedetomidine compared with intravenous dexmedetomidine administration. This result suggests that the topical application of dexmedetomidine may have a cough suppression effect, which is in accordance with 
other studies (27). To test whether dexmedetomidine combined with lidocaine had a significant effect on cough suppression, we set up the no dexmedetomidine group as a control; in that group, the incidence of moderate to severe coughing was $50 \%$. This finding may be related to the fact that dexmedetomidine can increase the effect of local anaesthetics (28). However, there was no significant improvement in glottis closure, complete jaw relaxation or limb movement in this study. This may be because dexmedetomidine has no muscle relaxant effects.

Previous studies have shown that intravenous use of dexmedetomidine causes intraoperative hypotension and bradycardia (29). In the present study, the dosages of ephedrine and atropine in the intravenous dexmedetomidine group were higher than those in the nebulized dexmedetomidine group and the no dexmedetomidine group. Therefore, the nebulized dexmedetomidine group had reduced haemodynamic changes. Ryu and colleagues found that dexmedetomidine was associated with a longer recovery time (5). In the present study, the recovery times of the nebulized dexmedetomidine group and no dexmedetomidine group were significantly shorter than those of the intravenous dexmedetomidine group.

The present study had the following limitations. First, given the small sample size in this study, changing only a few of the outcome data points might have changed the study conclusion. Second, the secondary endpoints may have been underpowered, and we were unable to detect adverse reactions in patients due to the case limitations. Third, although we used a similar dose of $0.6 \mu \mathrm{g} / \mathrm{kg}$ of dexmedetomidine for all cases, we did not measure the blood concentration of dexmedetomidine for technical reasons. The optimal concentration of nebulized dexmedetomidine inhalation should be further determined.

In summary, nebulized dexmedetomidine-lidocaine inhalation as a premedication for flexible bronchoscopy was well tolerated during bronchoscopies under moderate sedation and was associated with a reduced incidence of moderate to severe coughing, a shorter recovery time and reduced vasoconstrictor consumption. However, there were no significant differences in the rates of glottis closure, complete jaw relaxation and limb movement.

\section{Acknowledgments}

The authors would like to thank Jiayuan Sun, M.D., in the Department of Respiratory Therapy at Shanghai Chest Hospital, Shanghai, China for his suggestions and assistance with patient enrolment.

Funding: This work was supported by the Shanghai Municipal Commission of Health and Family Planning Project (201840319) and Project 81671078, supported by the National Natural Science Foundation of China.

\section{Footnote}

Conflicts of Interest: The authors have no conflicts of interest to declare.

Etbical Statement: The authors are accountable for all aspects of the work by ensuring that questions related to the accuracy or integrity of any part of the work are appropriately investigated and resolved. This study was approved by the Institutional Review Board of Shanghai Chest Hospital (IRB \#KS 1748), and written informed consent was obtained from each patient before enrolment.

\section{References}

1. Du Rand IA, Barber PV, Goldring J, et al. Summary of the British Thoracic Society guidelines for advanced diagnostic and therapeutic flexible bronchoscopy in adults. Thorax 2011;66:1014-5.

2. Lee K, Orme R, Williams D, et al. Prospective pilot trial of dexmedetomidine sedation for awake diagnostic flexible bronchoscopy. J Bronchology Interv Pulmonol 2010;17:323-8.

3. Kamibayashi T, Maze M. Clinical uses of alpha2adrenergic agonists. Anesthesiology 2000;93:1345-9.

4. Yuan F, Fu H, Yang P, et al. Dexmedetomidine-fentanyl versus propofol-fentanyl in flexible bronchoscopy: A randomized study. Exp Ther Med 2016;12:506-12.

5. Ryu JH, Lee SW, Lee JH, et al. Randomized double-blind study of remifentanil and dexmedetomidine for flexible bronchoscopy. Br J Anaesth 2012;108:503-11.

6. Ouchi K, Sugiyama K. Dexmedetomidine Dose Dependently Enhances the Local Anesthetic Action of Lidocaine in Inferior Alveolar Nerve Block: A Randomized Double-Blind Study. Reg Anesth Pain Med 2016;41:348-55.

7. Hetta DF, Kamal EE, Mahran AM, et al. Efficacy of local dexmedetomidine add-on for spermatic cord block anesthesia in patients undergoing intrascrotal surgeries: randomized controlled multicenter clinical trial. J Pain Res 2017;10:2621-8.

8. Mikami M, Zhang Y, Kim B, et al. Dexmedetomidine's 
inhibitory effects on acetylcholine release from cholinergic nerves in guinea pig trachea: a mechanism that accounts for its clinical benefit during airway irritation. BMC Anesthesiol 2017;17:52.

9. Zanaty OM, El Metainy SA. A comparative evaluation of nebulized dexmedetomidine, nebulized ketamine, and their combination as premedication for outpatient pediatric dental surgery. Anesth Analg 2015;121:167-71.

10. Pandey CK, Raza M, Ranjan R, et al. Intravenous lidocaine $0.5 \mathrm{mg} \cdot \mathrm{kg}-1$ effectively suppresses fentanyl-induced cough. Can J Anaesth 2005;52:172-5.

11. Du Rand IA, Blaikley J, Booton R, et al. British Thoracic Society guideline for diagnostic flexible bronchoscopy in adults: accredited by NICE. Thorax 2013;68 Suppl 1:i1-i44.

12. Ibrahim E, Sultan W, Helal S, et al. Pregabalin and dexmedetomidine conscious sedation for flexible bronchoscopy: a randomized double blind controlled study. Minerva Anestesiol 2019;85:487-93.

13. Riachy $M$, Khayat $G$, Ibrahim I, et al. A randomized double-blind controlled trial comparing three sedation regimens during flexible bronchoscopy: Dexmedetomidine, alfentanil and lidocaine. Clin Respir J 2018;12:1407-15.

14. Zhang H, Fang B, Zhou W. The efficacy of dexmedetomidine-remifentanil versus dexmedetomidinepropofol in children undergoing flexible bronchoscopy: A retrospective trial. Medicine (Baltimore) 2017;96:e5815.

15. Li X, Wang X, Jin S, et al. The safety and efficacy of dexmedetomidine-remifentanil in children undergoing flexible bronchoscopy: A retrospective dose-finding trial. Medicine (Baltimore) 2017;96:e6383.

16. Gao Y, Kang K, Liu H, et al. Effect of dexmedetomidine and midazolam for flexible fiberoptic bronchoscopy in intensive care unit patients: A retrospective study. Medicine (Baltimore) 2017;96:e7090.

17. Dich-Nielsen JO, Nagel P. Flexible fibreoptic bronchoscopy via the laryngeal mask. Acta Anaesthesiol Scand 1993;37:17-9.

18. Bauer TL, Berkheim DB. Bronchoscopy: Diagnostic and
Therapeutic for Non-Small Cell Lung Cancer. Surg Oncol Clin N Am 2016;25:481-91.

19. Chadha M, Kulshrestha M, Biyani A. Anaesthesia for bronchoscopy. Indian J Anaesth 2015;59:565-73.

20. Abdelmalak B, Khanna A, Tetzlaff J. Fospropofol, a new sedative anesthetic, and its utility in the perioperative period. Curr Pharm Des 2012;18:6241-52.

21. Dreher M, Cornelissen CG, Reddemann MA, et al. Nebulized versus Standard Local Application of Lidocaine during Flexible Bronchoscopy: A Randomized Controlled Trial. Respiration 2016;92:266-73.

22. Stolz D, Chhajed PN, Leuppi J, et al. Nebulized lidocaine for flexible bronchoscopy: a randomized, double-blind, placebo-controlled trial. Chest 2005;128:1756-60.

23. Sharma GK, Verma SP. Is Nebulized Lidocaine Adequate Topical Anesthesia for Diagnostic Transnasal Tracheoscopy? Ann Otol Rhinol Laryngol 2015;124:545-9.

24. Noitasaeng P, Vichitvejpaisal P, Kaosombatwattana U, et al. Comparison of Spraying and Nebulized Lidocaine in Patients Undergoing Esophago-Gastro-Duodenoscopy: A Randomized Trial. J Med Assoc Thai 2016;99:462-8.

25. Anttila M, Penttila J, Helminen A, et al. Bioavailability of dexmedetomidine after extravascular doses in healthy subjects. Br J Clin Pharmacol 2003;56:691-3.

26. Groeben H, Mitzner W, Brown RH. Effects of the alpha2-adrenoceptor agonist dexmedetomidine on bronchoconstriction in dogs. Anesthesiology 2004;100:359-63.

27. Mirkheshti A, Memary E, Honar BN, et al. The efficacy of local dexmedetomidine during fiberoptic nasotracheal intubation: A randomized clinical trial. J Anaesthesiol Clin Pharmacol 2017;33:209-14.

28. Liu Z, Jiang M, Xu T, et al. Analgesic effect of Ropivacaine combined with Dexmedetomidine on brachial plexus block. BMC Anesthesiol 2018;18:107.

29. Piao G, Wu J. Systematic assessment of dexmedetomidine as an anesthetic agent: a meta-analysis of randomized controlled trials. Arch Med Sci 2014;10:19-24.
Cite this article as: $\mathrm{Gu} \mathrm{W}, \mathrm{Xu} \mathrm{M}, \mathrm{Lu} \mathrm{H}$, Huang Q, Wu J. Nebulized dexmedetomidine-lidocaine inhalation as a premedication for flexible bronchoscopy: a randomized trial. J Thorac Dis 2019;11(11):4663-4670. doi: 10.21037/jtd.2019.10.59 